\author{
Charles L. Sprung \\ Katerina Rusinova \\ Otavio T. Ranzani
}

\section{Variability in forgoing life-sustaining treatments: reasons and recommendations}

Received: 21 April 2015

Accepted: 5 May 2015

Published online: 19 May 2015

(C) Springer-Verlag Berlin Heidelberg and ESICM 2015

\section{L. Sprung (ه)}

General Intensive Care Unit, Department of Anesthesiology and Critical Care Medicine, Hadassah Hebrew University Medical

Center, Jerusalem, Israel

e-mail: sprung@cc.huji.ac.il

\section{K. Rusinova}

Department of Anesthesiology and Intensive Care, 1st Faculty of Medicine, Institute for Medical Humanities, Charles University in Prague and General University Hospital, Prague, Czech Republic

\section{O. T. Ranzani}

Pulmonary Division, Respiratory Intensive Care Unit, Hospital das Clinicas, Heart Institute, University of Sao Paulo, Sao Paulo, Brazil

Over the last several decades physicians around the world have realized that not all critically ill patients can be saved and many should be allowed to die [1]. Life-sustaining therapies may be withheld or withdrawn or infrequently active shortening of the dying process occurs [2]. Despite different legal systems, attitudes, religions, cultures, and practices around the world, worldwide consensus was developed for the majority of key ethical issues regarding end-of-life practices [3]. The areas where consensus could not be obtained were withholding and withdrawing life-sustaining treatment and active shortening of the dying process [3].

Mark and colleagues [4] in this issue of Intensive Care Medicine systematically reviewed and found substantial variability in forgoing life-sustaining treatments between world regions, countries, individual ICUs within a country, and individual intensivists within one ICU. Withdrawal of life-sustaining treatment varied between 0 and $84 \%$. Interestingly, even patients admitted to ICUs with documented preferences for limited life-sustaining therapies such as donot-resuscitate (DNR) orders had wide variations in the proportions of patients who received cardiopulmonary resuscitation (CPR) or the reversal of treatment limitations [5]. Variability could be a marker of inappropriate treatment as reports of "too much care" are common.

What are the reasons for this variability in end-of-life therapies? Practices vary on the basis of geography $[1,2,6]$, religion [7, 8], culture [2], law [9], religiosity [8], patient [6], physician $[2,5,10]$, and institutional characteristics $[1,6$, 11], different attitudes between physicians and nurses, patients, and families [12], and triggers to limit treatments [10]. Is there any appropriate degree of variability? It will probably depend on the case mix of patients, their severity of illness, and differences between attitudes, religions, and cultures of caregivers and patients and their surrogates.

Another crucial question is the purpose for life-sustaining therapies? Answers differ for all involved in the decision. One explanation for variability might be different goals of therapy. Life-sustaining treatment options have recently been shown to be a "time-limited trial" followed by withdrawal and a known outcome of death or "open-ended" guided by physiologic objectives with the goal to avert death in the ICU [11]. Considering the complex interplay of processes concerning end-of-life including the variety of cultures worldwide and each patient's personal preferences, the high variability found by Mark et al. [4] is not unexpected. A portion of the variability could be explained by other factors. Some regions were not well represented in the review (e.g., Central/South America) which could represent selection bias introduced by language restriction. In addition, units involved in end-of-life research are more likely to publish ("publication bias"). 
We believe some variability is appropriate and a reflection of the human diversity of responses about decisions at the end-of-life. Variability in end-of-life care may reflect flexibility, attention, and sensitivity of physicians to subtle and unmeasured aspects of dying. Nevertheless, we must improve end-of-life care. There are problems with palliative care, communication, family satisfaction, and staff burnout. Trials to improve education/communication alone [13] and a program using selfefficacy theory focusing on staff were unable to improve end-of-life care [14]. The SUPPORT study demonstrated serious shortcomings in end-of-life care [13]. More than half of the physicians did not know when their patients preferred to avoid CPR, $46 \%$ of DNR orders were written within 2 days of death, and $50 \%$ of conscious patients dying in the hospital experienced moderate to severe pain half the time [13].

How can we improve? Patients, families, and even physicians have difficulties discussing end-of-life issues. First, we should improve awareness about end-of-life among doctors, patients, and families. This could help alleviate the fears and suffering of all involved in the decision-making process and avoid not providing the patient's wishes at the end-of-life. When the staff are not aware of end-of-life treatments and lack the required skills, the consequences can be catastrophic if discussions about end-of-life are not provided. Interventions for earlier education in end-of-life care are promising and should be tested [15]. It has been recently demonstrated that not only knowledge but also perceived self-efficacy or confidence plays a major role in the ability of physicians to discuss limitations at the end-of-life [16]. Doctors are less confident discussing DNR orders than medical procedures [16]. Regarding communication, improvements have occurred. Simple interventions (e.g., VALUE) [17] and structured approaches showed better outcomes [18]. Furthermore, organizational factors could provide better and friendlier environments to discuss end-of-life issues such as available rooms for family conferences and a multidisciplinary team.

As the authors point out, therapy withdrawal is usually documented whereas withholding which does not require an action may not be documented. Furthermore, as most country legislation has not addressed the issue of foregoing life support in the terminally ill, many doctors avoid discussing their actions or potentially incriminating documentation. Reliable data about limitations made outside the ICU on the wards are also lacking. Therefore, the incidence of both withholding and withdrawing treatments may be much higher than reported. As important as the authors' primary finding of variability of withdrawing therapy, withholding therapy is no less important. Withholding life-sustaining treatment also varied between 5 and $67 \%$.

Surprisingly and importantly, Mark et al. noted that DNR orders, probably the most frequent type of limitation, were defined variably as withdrawing, withholding, or as a separate limitation category and not withholding life-support. We agree with the authors' recommendation that future studies document the proportion of deaths preceded by a DNR order as a separate category within withholding life support so studies can be compared.

The topic of variability in forgoing life-sustaining treatments in the ICU is a complex issue involving multiple medical, social, religious, and cultural factors. Perhaps instead of focusing on variability as a target for interventions, we should study how various norms arise in the patient-physician-family relationship. Future studies should elucidate the reasons for the variability, develop means to reduce bad variation reflecting failure in professionalism and preserve good variation that makes care patient-centered [19], and differentiate those causes that are amenable to change (attitudes) and those that are not (religion and religiosity). While improved insights through continued high-quality research is necessary, it is also crucial to improve medical education to improve end-of-life management. There is also a great need to preserve dignity [20] and to develop mechanisms to measure the quality of end-of-life care.

Acknowledgments The authors thank Prof. Mayer Brezis who was extremely helpful in his review of the editorial and who made several important suggestions.

\section{References}

1. Azoulay E, Metnitz B, Sprung CL et al (2009) End-of-life practices in 282 intensive care units: data from the SAPS 3 database. Intensive Care Med 35:623-630

2. Sprung CL, Cohen SL, Sjokvist $P$ et al (2003) End-of-life practices in European intensive care units: the Ethicus Study. JAMA 290:790-797
3. Sprung CL, Truog RD, Curtis JR et al (2014) Seeking worldwide professional consensus on the principles of end-oflife care for the critically ill: the Welpicus study. Am J Respir Crit Care Med 190:855-866
4. Mark NM, Rayner SG, Lee, JR, Curtis JR (2015) Global variability in the withdrawal of life-sustaining treatment in the intensive care unit: a systematic review. Intensive Care Med [Epub ahead of print] 
5. Hart JL, Harhay MO, Gabler NB, Ratcliffe SJ, Quill CM, Halpern SD (2015) Variability among US intensive care units in managing the care of patients admitted with preexisting limits on life-sustaining therapies. JAMA Intern Med. doi:

10.1001/jamainternmed.2015.0372

6. Quill CM, Ratcliffe SJ, Harhay MO, Halpern SD (2014) Variation in decisions to forgo life-sustaining therapies in US ICUs. Chest 146:573-582

7. Sprung CL, Maia $\mathrm{P}$, Bulow $\mathrm{HH}$ et al (2007) The importance of religious affiliation and culture on end-of-life decisions in European intensive care units. Intensive Care Med 33:1732-1739

8. Bulow HH, Sprung CL, Baras M et al (2012) Are religion and religiosity important to end-of-life decisions and patient autonomy in the ICU? The Ethicatt study. Intensive Care Med 38:1126-1133

9. Luce JM, White DB (2009) A history of ethics and law in the intensive care unit. Crit Care Clin 25:221-237

10. Joynt GM, Lipman J, Hartog C et al (2015) The Durban World Congress Ethics Round Table IV: health care professional end-of-life decision making. J Crit Care 30:224-230
11. Barnato AE, Tate JA, Rodriguez KL, Zickmund SL, Arnold RM (2012) Norms of decision making in the ICU: a case study of two academic medical centers at the extremes of end-of-life treatment intensity. Intensive Care Med 38:1886-1896

12. Sprung CL, Carmel S, Baras $M$ et al (2007) Attitudes of European physicians, nurses, patients, and families regarding end-of-life decisions: the ETHICATT study. Intensive Care Med 33:104-110

13. The SUPPORT Principal Investigators (1995) A controlled trial to improve care for seriously ill hospitalized patients. The study to understand prognoses and preferences for outcomes and risks of treatments. JAMA 274:1591-1598

14. Curtis JR, Nielsen EL, Treece PD et al (2011) Effect of a quality-improvement intervention on end-of-life care in the intensive care unit: a randomized trial. Am J Respir Crit Care Med 183:348-355

15. Forte DN, Vincent JL, Velasco IT, Park M (2011) Association between education in EOL care and variability in EOL practice: a survey of ICU physicians. Intensive Care Med 38(404-412):24
16. Sulmasy DP, Sood JR, Ury WA (2008) Physicians' confidence in discussing do not resuscitate orders with patients and surrogates. J Med Ethics 34:96-101

17. Wysham NG, Mularski RA, Schmidt DM et al (2014) Long-term persistence of quality improvements for an intensive care unit communication initiative using the VALUE strategy. J Crit Care 29:450-454

18. Lautrette A, Darmon M, Megarbane B et al (2007) A communication strategy and brochure for relatives of patients dying in the ICU. N Engl J Med 356:469-478

19. Appleby J, Raleigh V, Frosini F, Bevan G, Gao H, Lyscom T (2011) Variations in health care. The good, the bad and the inexplicable. The King's Fund, London

20. Cook D, Rocker G (2014) Dying with dignity in the intensive care unit. N Engl J Med 370:2506-2514 